\title{
Erosão hídrica em diferentes sistemas de cultivo e níveis de cobertura do solo
}

\author{
Wilk Sampaio de Almeida(1), Daniel Fonseca de Carvalho(2), Elói Panachuki(3), \\ Wander Cardoso Valim ${ }^{(3)}$, Sonia Armbrust Rodrigues ${ }^{(3)}$ e Carlos Alberto Alves Varella(2)
}

\begin{abstract}
(1)Instituto Federal de Educação, Ciência e Tecnologia de Rondônia, Campus Colorado do Oeste, BR 435, km 63, CEP 76.993-000, Colorado do Oeste, RO, Brasil. E-mail: wilk.almeida@ifro.edu.br (2)Universidade Federal Rural do Rio de Janeiro, Departamento de Engenharia, BR-465, Km 7, CEP 23897-000 Seropédica, RJ, Brasil. E-mail: carvalho@ufrrj.br, varella@ufrrj.br (3)Universidade Estadual de Mato Grosso do Sul, Departamento de Agronomia, CEP 79200-000 Aquidauana, MS, Brasil. E-mail: eloip@uems.br, wander.cv@hotmail.com, soninha.agro@hotmail.com
\end{abstract}

Resumo - O objetivo deste trabalho foi avaliar, sob chuva simulada, o efeito da cobertura vegetal sobre a erosão de um Argissolo Vermelho, no Estado de Mato Grosso do Sul, nos seguintes sistemas de cultivo: soja, com preparo convencional; soja, em plantio direto; pastagem estabelecida, sem pisoteio animal; e condição de solo exposto, como padrão de comparação. A cada 20 dias, foram tiradas fotografias digitais para a estimativa da cobertura do solo, e chuvas simuladas de $60 \mathrm{~mm} \mathrm{~h}^{-1}$ foram aplicadas às parcelas experimentais, com uso do simulador portátil de chuvas. As perdas de solo, quantificadas a cada 2 min, variaram de 3,10 a $11,40 \mathrm{Mg} \mathrm{ha}^{-1}$, no solo exposto, e de 0,03 a $0,19 \mathrm{Mg} \mathrm{ha}^{-1}$ na pastagem; as perdas de água variaram de 300 a $555 \mathrm{~m}^{3} \mathrm{ha}^{-1}$, no solo exposto, e de 63 a $229 \mathrm{~m}^{3} \mathrm{ha}^{-1}$, no cultivo de soja sob plantio direto. As perdas de solo diminuíram com o aumento da cobertura vegetal. Na pastagem, a máxima cobertura foi de $98,10 \%$. Os efeitos benéficos do preparo convencional são temporários, o que torna o solo mais suscetível à erosão do que os sistemas conservacionistas.

Termos para indexação: Glycine max, Urochloa ruziziensis, perda de solo e água, chuva simulada.

\section{Hydraulic erosion in different tillage systems and soil cover}

\begin{abstract}
The objective of this work was to evaluate, under simulated rainfall, the effect of soil cover on the erosion of a Typic Hapludalf erosion, in the state of Mato Grosso do Sul, Brazil, in the following crop systems: soybean, under conventional tillage; soybean, under no-tillage; established pasture, without cattle trampling; and exposed soil, as a comparison standard. Digital photographs were taken every 20 days for soil cover estimate, and $60 \mathrm{~mm} \mathrm{~h}^{-1}$ simulated rainfall was applied on the experimental plots, using a portable sprinkler infiltrometer. Soil losses, quantified every $2 \mathrm{~min}$, ranged from 3.10 to $11.40 \mathrm{Mg} \mathrm{ha}^{-1}$, in exposed soil, and from 0.03 to $0.19 \mathrm{Mg} \mathrm{ha}^{-1}$, in established pasture; water losses ranged from 300 to $555 \mathrm{~m}^{3} \mathrm{ha}^{-1}$, in exposed soil, and from 63 to $229 \mathrm{~m}^{3} \mathrm{ha}^{-1}$, in soybean cultivation under no-tillage. Soil losses decreased with the increase in vegetation cover. In the pasture, the maximum vegetation cover was $98.10 \%$. Conventional tillage has temporary positive effects, which makes the soil more susceptible to erosion than the conservationist systems.
\end{abstract}

Index terms: Glycine max, Urochloa ruziziensis, soil and water losses, simulated rainfall.

\section{Introdução}

A erosão do solo é considerada um dos maiores problemas ambientais em escala global, pois, além de proporcionar perdas de solo e nutrientes, está associada a inundações, assoreamento e poluição de corpos hídricos (Wang et al., 2016). O processo erosivo é afetado por diferentes fatores, entre os quais a cobertura do solo e as práticas de manejo empregadas (Panagos et al., 2015).

Áreas com preparo convencional do solo e desprovidas de cobertura vegetal em superfície são mais suscetíveis à erosão hídrica, pois, este sistema favorece a formação do selamento superficial, caracterizado por uma fina camada de solo que se torna compactada pelo impacto direto da gota de chuva sobre o solo (Panachuki et al., 2011). No entanto, em sistemas considerados conservacionistas - como o plantio direto e a pastagem sob manejo adequado, com pouco ou nenhum revolvimento do solo e que mantenham cobertura vegetal sobre a superfície -, a formação de agregados maiores e mais estáveis é favorecida (Souza et al., 2005), o que proporciona aumento da resistência do solo à erosão (Engel et al., 2009).

De maneira isolada, a presença de resíduos vegetais como cobertura do solo é o fator mais importante na 
dissipação da energia de impacto das gotas da chuva (Cogo et al., 1984; Panachuki et al., 2011). Em áreas sob pastagem com alto percentual de cobertura, a rugosidade superficial do solo aumenta (Nacinovic et al., 2014), favorece maior infiltração de água e, consequentemente, menor escoamento supeficial (Amaral et al., 2008).

No Brasil, a pastagem ocupa uma área de 173 milhões de hectares, em que 117 milhões de hectares são de pastagens plantadas (Zimmer et al., 2012), dos quais $70 \%$ possuem algum tipo de degradação em estágio avançado (Censo..., 2007). A maior parte dessa área se encontra no bioma Cerrado, que é a principal região produtora de carne bovina no Brasil, com quase $34 \%$ do rebanho nacional, onde também predominam monocultivos de cana-de-açúcar e soja (Lapola et al., 2013). Nessa região, destaca-se o Estado do Mato Grosso do Sul, com 10,9\% do rebanho bovino e $6,9 \%$ da área com soja no Brasil (IBGE, 2015). Nos últimos anos, têm aumentado as áreas de exploração agropecuária sob sistemas conservacionistas na região, embora estudos sobre o efeito da cobertura vegetal e do preparo do solo no processo erosivo sejam, ainda, pouco expressivos.

Para ampliar a geração de informações referentes às perdas de solo e de água, em sistemas agrícolas sob diferentes tipos de preparo do solo e cobertura vegetal, a aplicação de chuvas simuladas vem sendo amplamente utilizada (Ries et al., 2009). O uso de simuladores de chuva portáteis possibilita a pesquisa, em campo, da dinâmica do processo erosivo alterada pelo manejo do solo, pois é de fácil repetibilidade e rapidez na obteção das taxas de erosão do solo (Alves Sobrinho et al., 2008).

O objetivo deste trabalho foi avaliar, sob chuva simulada, o efeito da cobertura vegetal sobre a erosão de um Argissolo Vermelho, no Estado de Mato Grosso do Sul, nos seguintes sistemas de cultivo: soja, com preparo convencional; soja, em plantio direto; pastagem estabelecida, sem pisoteio animal; e condição de solo exposto, como padrão de comparação.

\section{Material e Métodos}

O experimento foi realizado na Unidade Experimental da Universidade Estadual de Mato Grosso do Sul, em Aquidauana, MS, a $20^{\circ} 27^{\prime} \mathrm{S}$, $55^{\circ} 40^{\prime} \mathrm{W}$, a $191 \mathrm{~m}$ de altitude, de novembro/2013 a abril/2014. O solo da área experimental, classificado como Argissolo Vermelho distrófico típico (Schiavo et al., 2010), apresentava areia, silte e argila a 770, 110 e $120 \mathrm{~g} \mathrm{~kg}^{-1}$ - na camada $0-24 \mathrm{~cm}$-, e 610,140 e 250 $\mathrm{g} \mathrm{kg}^{-1}$ - na camada 50-65 $\mathrm{cm}-$, respectivamente. O clima regional é do tipo Aw (tropical úmido), segundo a classificação de Köppen-Geiger. Os valores médios anuais de temperatura e precipitação são de $24^{\circ} \mathrm{C}$ e $1.400 \mathrm{~mm}$, respectivamente, de acordo com Santos et al. (2014). A declividade média da área experimental é $0,03 \mathrm{~m} \mathrm{~m}^{-1}$.

O delineamento experimental inteiramente casualizado foi adotado, com quatro tratamentos e quatro repetições, em arranjo de parcelas subdivididas no tempo. Os tratamentos foram caracterizados por culturas associadas aos seguintes sistemas de preparo do solo: soja (Glycine max L.) cultivada com preparo convencional (PC), na direção do declive; soja cultivada em sistema plantio direto (PD); pastagem estabelecida (PA) com Urochloa ruziziensis, sem pisoteio animal, e com um ano desde a data de plantio; e solo exposto (SE), com preparo convencional na direção do declive e mantido sem vegetação. Portanto, nas parcelas, avaliaram-se os tipos de cobertura do solo (SE, PC, PD e PA) e, nas subparcelas, as épocas de aplicação das chuvas simuladas aos 0, 20, 40, 60, 80 e 100 dias após a semeadura (DAS).

O preparo convencional do solo foi caracterizado por uma gradagem aradora, seguida de duas gradagens niveladoras/destorroadoras. A cultivar de soja utilizada foi a 'SYN9074 RR', e a adubação de plantio consistiu de $300 \mathrm{~kg} \mathrm{ha}^{-1}$ da formulação $\mathrm{N}-\mathrm{P}_{2} \mathrm{O}_{5}-\mathrm{K}_{2} \mathrm{O}$ 0-20-20.

$\mathrm{O}$ simulador de chuvas utilizado, desenvolvido por Alves Sobrinho et al. (2008), foi calibrado para aplicar chuvas com intensidade de $60 \mathrm{~mm} \mathrm{~h}^{-1}$, valor adotado em estudos desta natureza (Panachuki et al., 2011; Huang et al., 2013). A parcela-teste que recebeu a precipitação apresenta área útil de $0,70 \mathrm{~m}^{2}$, e é delimitada por chapas de aço galvanizado. Em uma das laterais, há um bico em formato de funil, para coleta do volume de água escoado em superfície. Os delimitadores das parcelas permaneceram fixos na área, durante todo o período de avaliação correspondente ao ciclo da soja.

A rugosidade da superfície do solo foi estimada com uso do rugosímetro de varetas, acoplado a uma máquina fotográfica digital, conforme descrito por Panachuki et al. (2010). O equipamento é constituído de 20 varetas de alumínio, alinhadas e distanciadas

Pesq. agropec. bras., Brasília, v.51, n.9, p.1110-1119, set. 2016 DOI: $10.1590 / \mathrm{S} 0100-204 X 2016000900010$ 
$30 \mathrm{~mm}$ entre si, que foram deslocadas no sentido do aclive, tendo ocupado 20 posições em cada parcela. Em cada posição, foi feito um registro fotográfico das 20 varetas verticais, o que gerou um grid de $0,36 \mathrm{~m}^{2}$, com imagens das alturas de 400 varetas, para cada momento avaliado, em cada parcela experimental. Cada fotografia foi digitalizada por meio de programa computacional, e a quantidade de pixels de cada vareta foi avaliada, com sua posterior transformação em unidade de comprimento correspondente à altura de cada vareta. Em seguida, estimou-se a rugosidade ao acaso (RR), por meio do desvio-padrão das alturas do microrrelevo, sem transformação logarítmica e sem eliminar os valores extremos (10\% superiores e $10 \%$ inferiores), para a caracterização da rugosidade da superfície do solo. Esse procedimento foi feito em três momentos, nos tratamentos solo exposto e soja cultivada sob preparo convencional, antes e após as operações de preparo do solo e após a sexta aplicação de chuva simulada. Nos demais tratamentos, foram feitas duas avaliações: inicial e final (antes e após a aplicação das chuvas), em razão do não revolvimento do solo para este estudo. Embora a análise da rugosidade seja feita considerando-se a superfície do solo, no tratamento PA a avaliação foi feita com a cultura que cobria o solo.

Após a sexta etapa de aplicação das chuvas, em cada área experimental $\left(0,70 \mathrm{~m}^{2}\right)$, coletaram-se amostras de solo, das camadas $0-10,10-20$ e $20-40 \mathrm{~cm}$, para a caracterização da densidade, porosidade e estabilidade de agregados do solo, conforme Claessen (1997). Das mesmas profundidades, também se avaliaram: a umidade do solo antecedente à chuva simulada, estimada pelo método gravimétrico; e a resistência do solo à penetração, quantificada em laboratório com penetrógrafo digital de bancada (Serafim et al., 2008), com três leituras por amostra indeformada. O tempo para escoamento foi considerado do início da aplicação da chuva até a constatação do escoamento em superfície.

Para avaliação da cobertura vegetal de cada parcela experimental, tomaram-se fotografias digitais em cada época de avaliação, antes da aplicação de chuvas. A aquisição das imagens ocorreu no período do dia entre $11 \mathrm{e} 12 \mathrm{~h}$, à altura de $1,50 \mathrm{~m}$ em relação à superfície do solo, com foco na área de $0,70 \mathrm{~m}^{2}$ e zoom de $1.4 \mathrm{x}$. A estimativa da cobertura do solo, denominada índice de cobertura vegetal (ICV), foi realizada por meio do algoritmo Serobin, implementado no programa computacional Matlab (Cruz et al., 2008). Para a utilização do algoritmo, as imagens foram recortadas e, em seguida, identificaram-se 10 pontos representativos do atributo planta e 10 pontos do atributo solo, em uma imagem-base para cada tratamento, que foi utilizada para a classificação das demais imagens. No tratamento $\mathrm{PD}$, considerou-se a soja e a palhada da cultura anterior (milho, Zea mays) como mesma cobertura.

Antes da aplicação das chuvas, aos 0 e 60 DAS, uniformizou-se a umidade do solo, por meio do molhamento das parcelas com um sistema de gotejamento, o que constitui pré-requisito à aplicação da chuva artificial (Cogo et al., 1984). Nas demais épocas, este procedimento ocorreu por chuva natural. Após esta etapa, iniciaram-se os testes com o simulador, que tiveram duração de 60 min a partir do início do escoamento superficial (ES). Após o início do ES, o volume escoado era coletado durante $2 \mathrm{~min}$, tendo sido obtidas, portanto, 30 amostras em cada ensaio com o simulador. Em laboratório, quantificou-se a perda de solo e de água de cada teste, conforme Panachuki et al. (2011).

Os valores de rugosidade da superfície do solo, resistência do solo à penetração, propriedades físicas do solo, umidade do solo antecedente à aplicação das chuvas, cobertura vegetal, tempo para início do escoamento e perdas de solo $\left(\mathrm{Mg} \mathrm{ha}^{-1}\right)$ e de água $\left(\mathrm{m}^{3} \mathrm{ha}^{-1}\right)$ foram submetidos à análise de variância, e as médias entre os níveis do fator tipo de cobertura foram comparadas pelo teste de Scott-Knott, a $5 \%$ de probabilidade.

\section{Resultados e Discussão}

A rugosidade ao acaso (RR) diferiu entre os sistemas solo exposto (SE) e soja sob preparo convencional, antes do preparo do solo, graças ao resíduo vegetal de milho cultivado anteriormente na área de PC. Após o preparo do solo, os valores da RR em SE e PC foram semelhantes, em razão da homogeneização promovida pelo uso da grade aradora e da grade niveladora. Entretanto, após a aplicação das chuvas, a RR em SE diminuiu, pois o impacto das gotas de chuva e a enxurrada reduziram a rugosidade da superfície desprovida de cobertura vegetal, conforme também descrito por Dalla Rosa et al. (2012). Em PC, por sua vez, a RR foi semelhante à encontrada em $\mathrm{PD}$, em razão dos restos vegetais da soja dispostos sobre o solo, que 
promovem, entretanto, maior resistência às alterações promovidas pela chuva do que na condição de preparo do solo. No sistema plantio direto, o valor inicial elevado da RR resultou da presença de restos vegetais do milho, e o valor final, da palhada da soja associada à do milho. Na pastagem, a RR final foi menor do que a inicial, como consequência da aplicação das chuvas, que tombaram a pastagem (Tabela 1).

Verificou-se diferença entre os tratamentos quanto à densidade e à porosidade do solo (macro, micro e total) (Tabela 2). Para a camada superficial (0-10 $\mathrm{cm}$ ), na pastagem (PA), a maior macroporosidade

Tabela 1. Rugosidade ao acaso da superfície do solo, por sistema de cultivo e por época ${ }^{(1)}$.

\begin{tabular}{|c|c|c|c|c|c|}
\hline \multirow[t]{2}{*}{ Época } & \multicolumn{4}{|c|}{ Rugosidade ao acaso (RR, mm) } & \multirow{2}{*}{$\begin{array}{l}\text { Média } \\
\text { Geral }\end{array}$} \\
\hline & SE & $\mathrm{PC}$ & PD & PA & \\
\hline Antes do preparo do solo (PS) & $2,29 \mathrm{bB}$ & $10,44 \mathrm{aA}$ & $-(2)$ & $-(2)$ & $6,37 \mathrm{~B}$ \\
\hline Após o PS e antes da aplicação das chuvas & $6,44 \mathrm{cA}$ & $6,46 \mathrm{cB}$ & $13,37 \mathrm{bA}$ & $67,29 \mathrm{aA}$ & $23,39 \mathrm{~A}$ \\
\hline Depois da aplicação das chuvas & $1,24 \mathrm{cB}$ & $9,62 \mathrm{bA}$ & $9,36 \mathrm{bA}$ & $56,34 \mathrm{aB}$ & $19,14 \mathrm{~A}$ \\
\hline
\end{tabular}

${ }^{(1)}$ Médias seguidas de letras iguais, minúsculas nas linhas e maiúsculas nas colunas, não diferem pelo teste de Scott-Knott, a 5\% de probabilidade. ${ }^{(2)}$ Não houve preparo do solo. SE, solo exposto; PC, soja cultivada, com preparo convencional do solo; PD, soja cultivada em plantio direto; PA, pastagem estabelecida, sem pisoteio animal.

Tabela 2. Propriedades físicas do Argissolo Vermelho distrófico, por camadas e por sistemas de cultivo ${ }^{(1)}$.

\begin{tabular}{|c|c|c|c|c|c|}
\hline Camada $(\mathrm{cm})$ & SE & $\mathrm{PC}$ & $\mathrm{PD}$ & $\mathrm{PA}$ & Média geral \\
\hline \multicolumn{6}{|c|}{ Macroposidade (\%) } \\
\hline $0-10$ & $17,15 \mathrm{bA}$ & $18,47 \mathrm{aA}$ & $14,16 \mathrm{bA}$ & $21,67 \mathrm{aA}$ & $17,86 \mathrm{~A}$ \\
\hline $10-20$ & $10,93 \mathrm{aB}$ & $11,13 \mathrm{aB}$ & $13,96 \mathrm{aA}$ & $9,92 \mathrm{aB}$ & $11,48 \mathrm{~B}$ \\
\hline $20-40$ & $8,55 \mathrm{aC}$ & $8,45 \mathrm{aB}$ & $9,03 \mathrm{aB}$ & $8,15 \mathrm{aB}$ & $8,54 \mathrm{C}$ \\
\hline \multicolumn{6}{|c|}{ Microposidade (\%) } \\
\hline $0-10$ & $18,73 \mathrm{aB}$ & $16,85 \mathrm{aB}$ & $18,74 \mathrm{aA}$ & $17,77 \mathrm{aA}$ & $18,02 \mathrm{~B}$ \\
\hline $10-20$ & $20,50 \mathrm{aA}$ & $19,14 \mathrm{aA}$ & $17,82 \mathrm{aA}$ & $19,26 \mathrm{aA}$ & $19,18 \mathrm{~A}$ \\
\hline $20-40$ & $19,87 \mathrm{aA}$ & $19,86 \mathrm{aA}$ & $19,25 \mathrm{aA}$ & $17,96 \mathrm{aA}$ & $19,23 \mathrm{~A}$ \\
\hline \multicolumn{6}{|c|}{ Porosidade total $(\%)$} \\
\hline $0-10$ & $35,88 \mathrm{bA}$ & $35,33 \mathrm{bA}$ & $32,90 \mathrm{bA}$ & $39,43 \mathrm{aA}$ & $35,89 \mathrm{~A}$ \\
\hline $10-20$ & $31,43 \mathrm{aB}$ & $30,27 \mathrm{aB}$ & $31,78 \mathrm{aA}$ & $29,17 \mathrm{aB}$ & $30,66 \mathrm{~B}$ \\
\hline $20-40$ & $28,41 \mathrm{aB}$ & $28,30 \mathrm{aB}$ & $28,28 \mathrm{aB}$ & $26,10 \mathrm{aC}$ & $27,77 \mathrm{C}$ \\
\hline \multicolumn{6}{|c|}{ C orgânico $\left(\mathrm{g} \mathrm{kg}^{-1}\right)$} \\
\hline $0-10$ & $42,25 \mathrm{aA}$ & $31,33 \mathrm{bA}$ & $29,80 \mathrm{bA}$ & $36,48 \mathrm{aA}$ & $34,96 \mathrm{~A}$ \\
\hline $10-20$ & $19,57 \mathrm{bB}$ & $31,44 \mathrm{aA}$ & $19,46 \mathrm{bB}$ & $18,78 \mathrm{bB}$ & $22,31 \mathrm{~B}$ \\
\hline $20-40$ & $17,53 \mathrm{aB}$ & $18,10 \mathrm{aB}$ & $16,51 \mathrm{aB}$ & $14,81 \mathrm{aB}$ & $16,74 \mathrm{C}$ \\
\hline \multicolumn{6}{|c|}{ Densidade do solo $\left(\mathrm{Mg} \mathrm{m}^{-3}\right)$} \\
\hline $0-10$ & $1,43 \mathrm{bB}$ & $1,39 \mathrm{bB}$ & $1,54 \mathrm{aB}$ & $1,30 \mathrm{cC}$ & $1,42 \mathrm{C}$ \\
\hline $10-20$ & $1,59 \mathrm{aA}$ & $1,55 \mathrm{aA}$ & $1,57 \mathrm{aB}$ & $1,60 \mathrm{aB}$ & $1,58 \mathrm{~B}$ \\
\hline $20-40$ & $1,66 \mathrm{aA}$ & $1,61 \mathrm{aA}$ & $1,65 \mathrm{aA}$ & $1,69 \mathrm{aA}$ & $1,65 \mathrm{~A}$ \\
\hline \multicolumn{6}{|c|}{ Diâmetro médio geométrico dos agregados (DMG, mm) } \\
\hline $0-10$ & $1,14 \mathrm{bA}$ & $1,15 \mathrm{bA}$ & $1,29 \mathrm{bA}$ & $2,55 \mathrm{aA}$ & $1,54 \mathrm{~A}$ \\
\hline $10-20$ & $1,14 \mathrm{aA}$ & $1,50 \mathrm{aA}$ & $0,90 \mathrm{aA}$ & $1,30 \mathrm{aB}$ & $1,21 \mathrm{~B}$ \\
\hline $20-40$ & $0,84 \mathrm{aA}$ & $0,78 \mathrm{aA}$ & $0,79 \mathrm{aA}$ & $0,95 \mathrm{aB}$ & $0,84 \mathrm{C}$ \\
\hline \multicolumn{6}{|c|}{ Diâmetro médio ponderado dos agregados (DMP, mm) } \\
\hline$\overline{0-10}$ & $1,91 \mathrm{bA}$ & $2,07 \mathrm{bA}$ & $2,26 \mathrm{bA}$ & $3,44 \mathrm{aA}$ & $2,42 \mathrm{~A}$ \\
\hline $10-20$ & $1,92 \mathrm{aA}$ & $2,48 \mathrm{aA}$ & $1,46 \mathrm{aB}$ & $2,07 \mathrm{aB}$ & $1,98 \mathrm{~B}$ \\
\hline $20-40$ & $1,15 \mathrm{aA}$ & $1,30 \mathrm{aB}$ & $1,07 \mathrm{aB}$ & $1,47 \mathrm{aB}$ & $1,25 \mathrm{C}$ \\
\hline \multicolumn{6}{|c|}{ Resistência do solo à penetração (RP, $\mathrm{MPa})$} \\
\hline $0-10$ & $0,32 \mathrm{bC}$ & $0,44 \mathrm{bB}$ & $0,99 \mathrm{aA}$ & $0,42 \mathrm{bB}$ & $0,54 \mathrm{~B}$ \\
\hline $10-20$ & $0,91 \mathrm{aB}$ & $1,07 \mathrm{aA}$ & $0,77 \mathrm{aA}$ & $1,35 \mathrm{aA}$ & $1,03 \mathrm{~A}$ \\
\hline $20-40$ & $1,43 \mathrm{aA}$ & $1,00 \mathrm{aA}$ & $1,03 \mathrm{aA}$ & $1,37 \mathrm{aA}$ & $1,21 \mathrm{~A}$ \\
\hline
\end{tabular}

(1)Médias seguidas de letras iguais, minúsculas nas linhas e maiúsculas nas colunas, não diferem pelo teste de Scott-Knott, a 5\% de probabilidade. SE, solo exposto; PC, soja cultivada, com preparo convencional do solo; PD, soja cultivada em plantio direto; PA, pastagem estabelecida, sem pisoteio animal. 
está relacionada, possivelmente, ao teor de matéria orgânica proveniente do sistema radicular das plantas (Prado et al., 2014). Para a maioria das culturas, o limite crítico da densidade do solo pode variar, tendo sido relatados valores de 1,45 $\mathrm{Mg} \mathrm{m}^{-3}$ (Corsini \& Ferraudo, 1999) a $1,75 \mathrm{Mg} \mathrm{m}^{-3}$ (Reinert et al., 2008). Portanto, a densidade do solo não restringiu o desenvolvimento radicular, e os maiores valores em profundidade podem estar associados ao adensamento natural do horizonte subsuperficial do solo. As camadas com elevada densidade e baixa macroporosidade do solo favorecem a erosão hídrica e limitam o crescimento de raízes (Corsini \& Ferraudo, 1999).

$\mathrm{Na}$ pastagem, os maiores diâmetros médios geométricos (DMG) e ponderados (DMP) dos agregados tornam este sistema mais resistente à desagregação do solo (Prado et al., 2014). No entanto, em SE, PC e PD, os valores de DMP foram semelhantes. Assim, fica evidenciado o efeito da estabilidade dos agregados (DMG e DMP) na estrutura do solo, sobretudo nos sistemas conservacionistas (PD e PA), em que a matéria orgânica contribui com a formação de agregados maiores e mais estáveis (Souza et al., 2005; Prado et al., 2014). A estabilidade de agregados diminuiu com o aumento da profundidade (Souza et al., 2005), em virtude do maior teor de matéria orgânica na camada superficial, proveniente do sistema radicular das plantas (Prado et al., 2014), associado à maior densidade do solo e ao maior conteúdo de argila na camada subsuperficial.

A resistência do solo à penetração aumentou com a profundidade e, com exceção do $\mathrm{SE}$, não houve diferença entre as camadas 10-20 e $20-40 \mathrm{~cm}$, possivelmente decorrente de menor macroporosidade nessas camadas (Serafim et al., 2008) e da camada subsuperficial adensada nos Argissolos. Além disso, a acomodação das partículas do solo após o preparo e a ausência de cobertura vegetal, em SE, intensificam o efeito do impacto direto das gotas de chuva. Entretanto, a resistência à penetração foi maior na camada superficial do plantio direto, decorrente do não revolvimento do solo (Reinert et al., 2008).

A umidade do solo antecedente aos testes de campo foi semelhante entre as camadas avaliadas em cada tratamento. Contudo, nos sistemas PC (20 e 40 DAS) e PA (20 DAS e 80 DAS), a umidade na camada superficial foi menor do que nas demais camadas (Tabela 3), possivelmente, em virtude da água retida nos microporos (Cogo et al., 1984).
Nas imagens binárias classificadas para estimativa do ICV, a cor branca representa o atributo planta e a cor preta o atributo solo (Figura 1). No SE, a cobertura vegetal foi igual a zero, durante todo o estudo, o que o caracteriza como o mais suscetível ao processo erosivo, em comparação aos demais tratamentos. No entanto, nos tratamentos PC e PD, a cobertura vegetal aumentou, da primeira à última avaliação, em razão do desenvolvimento e crescimento da soja. No tratamento PA, a cobertura se manteve elevada e semelhante, ao longo do período de avaliação. Em geral, a pastagem apresentou maior cobertura vegetal, com ICV de 74,9 a $98,1 \%$. Apenas nas épocas 60 e 80 DAS, o ICV foi 8,06 e $12,30 \%$ menor, respectivamente, do que no sistema convencional de cultivo de soja, em que variou de zero a $93,75 \%$ (Figura 2 A). No cultivo de soja sob plantio direto, constatou-se ICV de 37,37 a 84,50\%, aos 40 DAS. Neste sistema, o ICV foi menor do que aquele obtido no sistema convencional de cultivo 34,52 e 65,65\% -, respectivamente, aos 60 e 80 DAS. Entretanto, aos 0 e 20 DAS, o ICV do PD foi 3,7 e 2,8 vezes maior do que no sistema convencional, em consequência dos resíduos da cultura anterior, o que resultou em menor desagregação e perdas de solo (Engel et al., 2009). A cobertura vegetal associada ao não revolvimento do solo torna este sistema mais resistente ao processo erosivo. Aos $100 \mathrm{DAS}$, o ICV nos sistemas soja sob preparo convencional e soja sob plantio direto diminuiu, graças à dessecação química da cultura, no entanto, foi maior do que $50 \%$, pois a cultura não havia sido colhida no momento da última avaliação.

O tempo para início do escoamento (TES) em superfície diferiu entre os tratamentos PA e PD. Na área de pastagem, o TES aumentou ao longo do período de avaliação e foi maior do que nos demais sistemas, em todas as épocas, provavelmente em razão do maior incremento de biomassa desta cultura. Nos demais sistemas, o TES diminuiu ao longo das avaliações, sobretudo em consequência do selamento superficial. Apenas na avaliação correspondente aos 60 DAS, o TES do PD diferiu daquele do SE e do PC, embora o tempo em PD tenha sido numericamente maior do que nos demais sistemas (Figura 2 B). Constatouse, ainda, aos 40 DAS, o aumento do TES no PC e $\mathrm{PD}$, ocasionados pelo desenvolvimento da soja, pois, isoladamente, a cobertura vegetal evita a desagregação das partículas do solo, o que é um importante fator na 
dissipação da energia do impacto das gotas da chuva (Panachuki et al., 2011). As diferenças dos valores de TES, entre os sistemas avaliados, estão relacionadas ao tipo e quantidade de cobertura vegetal sobre a superfície do solo (Cogo et al., 1984) que, no PC, PD e PA aumentam com o desenvolvimento e crescimento das culturas, além da interação das condições de superfície do terreno com as propriedades físicas do solo.

As menores perdas de solo ocorreram em PD e em PA, e o menor valor foi medido aos 60 DAS no PD. Aos 80 DAS, a acentuada redução das perdas de solo decorreu da maior cobertura vegetal proporcionada pela soja. O aumento das perdas de solo aos $100 \mathrm{DAS}$, em relação aos 80 DAS, pode ser justificado pela menor cobertura do solo em razão da dessecação da cultura da soja. Em PD e em PA, as menores perdas de solo resultam da elevada cobertura vegetal das plantas e dos resíduos vegetais da cultura antecessora sobre a superfície do solo (Engel et al., 2009) em PD, que atuam como barreira física ao impacto da gota de chuva, além da manutenção do teor de umidade do solo e retenção de água nas microdepressões (Cogo et al., 1984), o que aumenta a infiltração de água no solo e diminui o desprendimento e transporte de partículas pelo escoamento superficial (Figura $3 \mathrm{~A}$ ).

As perdas de solo observadas em SE, aos 40 e 100 DAS, superaram, respectivamente, em 82,5 e 168,8 vezes aquelas observadas no PA, e em 46,3 e 16,2 vezes as observadas em PD. O padrão de perda de solo no SE foi crescente, pois ocorreu maior selamento da superfície do solo e consequente diminuição da rugosidade superficial ao longo das avaliações. Além da baixa rugosidade, o teor de umidade armazenada no solo, no momento antecedente à aplicação da chuva artificial, favoreceu a desagregação das partículas do solo e proporcionou a maior geração de sedimentos aos $40 \mathrm{DAS}$, no total de $11,4 \mathrm{Mg} \mathrm{ha}^{-1}$. Nas etapas seguintes, ocorreu diminuição da perda de solo, porém, aos 100 DAS houve aumento de perda de solo, provavelmente decorrente do efeito combinado da energia cinética da chuva, e da menor rugosidade e do

Tabela 3. Umidade do solo anterior à aplicação das chuvas, nos sistemas de cultivo por camadas e por época ${ }^{(1)}$.

\begin{tabular}{|c|c|c|c|c|c|}
\hline \multirow{2}{*}{$\begin{array}{l}\text { Camada } \\
(\mathrm{cm})\end{array}$} & \multicolumn{4}{|c|}{ Umidade anterior à chuva $\left(\mathrm{cm}^{3} \mathrm{~cm}^{-3}\right)$} & \multirow{2}{*}{$\begin{array}{l}\text { Média } \\
\text { geral }\end{array}$} \\
\hline & SE & $\mathrm{PC}$ & $\mathrm{PD}$ & PA & \\
\hline & \multicolumn{5}{|c|}{0 DAS } \\
\hline $0-10$ & $24,62 \mathrm{aA}$ & $20,72 \mathrm{aA}$ & $17,30 \mathrm{bA}$ & $22,51 \mathrm{aA}$ & $21,29 \mathrm{~A}$ \\
\hline $10-20$ & $22,56 \mathrm{aA}$ & $23,17 \mathrm{aA}$ & $17,99 \mathrm{bA}$ & $21,88 \mathrm{aA}$ & $21,39 \mathrm{~A}$ \\
\hline \multirow[t]{2}{*}{$20-40$} & $21,25 \mathrm{aA}$ & $20,30 \mathrm{aA}$ & $17,77 \mathrm{aA}$ & $24,50 \mathrm{aA}$ & $20,95 \mathrm{~A}$ \\
\hline & \multicolumn{5}{|c|}{$20 \mathrm{DAS}$} \\
\hline $0-10$ & $12,11 \mathrm{aA}$ & $12,44 \mathrm{aA}$ & $15,75 \mathrm{aA}$ & $12,89 \mathrm{aA}$ & $13,29 \mathrm{~A}$ \\
\hline 10-20 & $14,96 \mathrm{aA}$ & $15,74 \mathrm{aB}$ & $16,59 \mathrm{aA}$ & $19,03 \mathrm{aB}$ & $16,58 \mathrm{~B}$ \\
\hline \multirow[t]{2}{*}{$20-40$} & $15,92 \mathrm{aA}$ & $17,78 \mathrm{aB}$ & $17,36 \mathrm{aA}$ & $17,15 \mathrm{aB}$ & $17,05 \mathrm{~B}$ \\
\hline & \multicolumn{5}{|c|}{$40 \mathrm{DAS}$} \\
\hline $0-10$ & $11,82 \mathrm{aA}$ & $6,77 \mathrm{bA}$ & $11,63 \mathrm{aA}$ & $12,01 \mathrm{aA}$ & $10,57 \mathrm{~A}$ \\
\hline $10-20$ & $13,27 \mathrm{aA}$ & $9,64 \mathrm{aB}$ & $12,51 \mathrm{aA}$ & $15,24 \mathrm{aA}$ & $12,66 \mathrm{~B}$ \\
\hline \multirow[t]{2}{*}{$\underline{20-40}$} & $15,16 \mathrm{aA}$ & $12,43 \mathrm{aB}$ & $15,25 \mathrm{aA}$ & $16,06 \mathrm{aA}$ & $14,72 \mathrm{C}$ \\
\hline & \multicolumn{5}{|c|}{$60 \mathrm{DAS}$} \\
\hline $0-10$ & $8,54 \mathrm{aA}$ & $4,61 \mathrm{aA}$ & $6,20 \mathrm{aA}$ & $8,81 \mathrm{aA}$ & $7,04 \mathrm{~A}$ \\
\hline $10-20$ & $10,31 \mathrm{aA}$ & $4,56 \mathrm{bA}$ & $6,47 \mathrm{bA}$ & $8,70 \mathrm{aA}$ & $7,51 \mathrm{~A}$ \\
\hline \multirow[t]{2}{*}{$20-40$} & $13,01 \mathrm{bA}$ & $4,51 \mathrm{aA}$ & 7,02aA & $6,90 \mathrm{aA}$ & $7,86 \mathrm{~A}$ \\
\hline & \multicolumn{5}{|c|}{80 DAS } \\
\hline $0-10$ & $14,60 \mathrm{aA}$ & $8,09 \mathrm{bA}$ & $13,81 \mathrm{aA}$ & $11,84 \mathrm{aA}$ & $12,08 \mathrm{~A}$ \\
\hline 10-20 & $15,66 \mathrm{aA}$ & $10,53 \mathrm{bA}$ & $15,77 \mathrm{aA}$ & $16,25 \mathrm{aB}$ & $14,55 \mathrm{~B}$ \\
\hline \multirow[t]{2}{*}{$20-40$} & $15,12 \mathrm{aA}$ & $10,95 \mathrm{bA}$ & $16,90 \mathrm{aA}$ & $17,73 \mathrm{aB}$ & $15,17 \mathrm{~B}$ \\
\hline & \multicolumn{5}{|c|}{100 DAS } \\
\hline $0-10$ & $13,23 \mathrm{aA}$ & $13,08 \mathrm{aA}$ & $22,56 \mathrm{bA}$ & $16,30 \mathrm{aA}$ & $16,29 \mathrm{~A}$ \\
\hline $10-20$ & $15,58 \mathrm{aA}$ & $12,63 \mathrm{aA}$ & $22,85 \mathrm{bA}$ & $20,29 \mathrm{bA}$ & $16,86 \mathrm{~A}$ \\
\hline $20-40$ & $15,87 \mathrm{aA}$ & $13,97 \mathrm{aA}$ & $19,43 \mathrm{bA}$ & $18,17 \mathrm{bA}$ & $17,83 \mathrm{~A}$ \\
\hline
\end{tabular}

${ }^{(1)}$ Médias seguidas de letras iguais, minúsculas nas linhas e maiúsculas nas colunas, não diferem pelo teste de Scott-Knott, a 5\% de probabilidade. SE, solo exposto; PC, soja cultivada sob preparo convencional do solo; PD, soja cultivada, com plantio direto; e PA, pastagem estabelecida, sem pisoteio animal. 

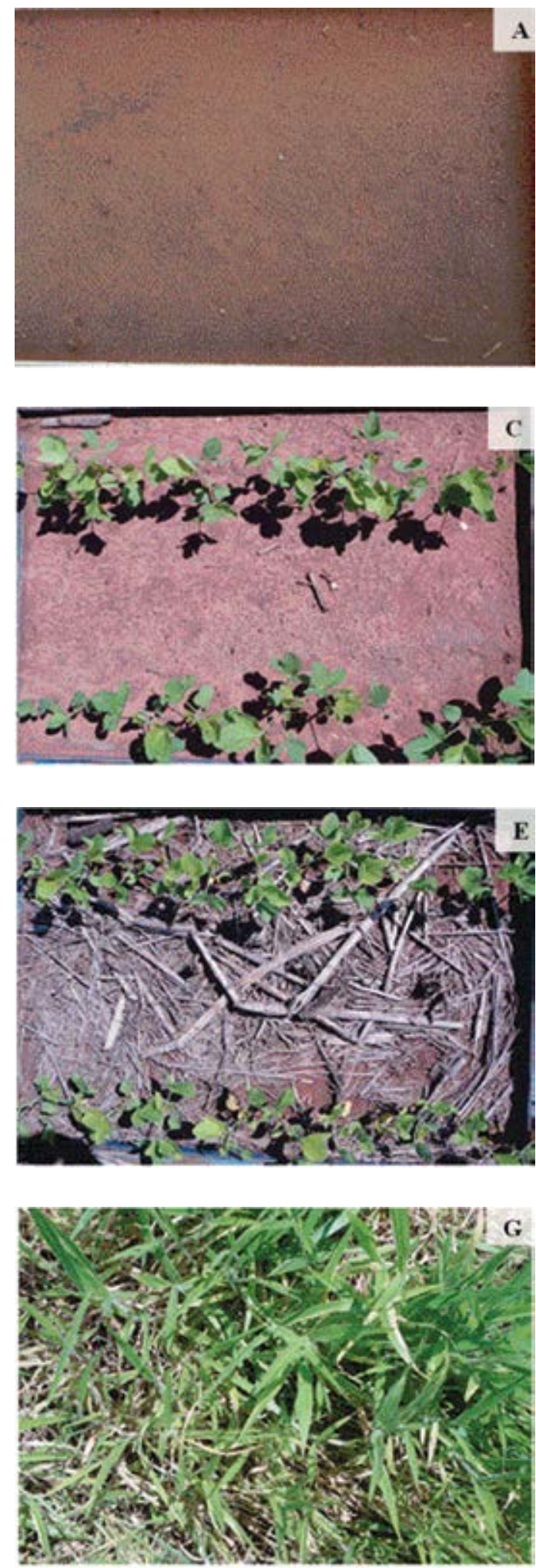
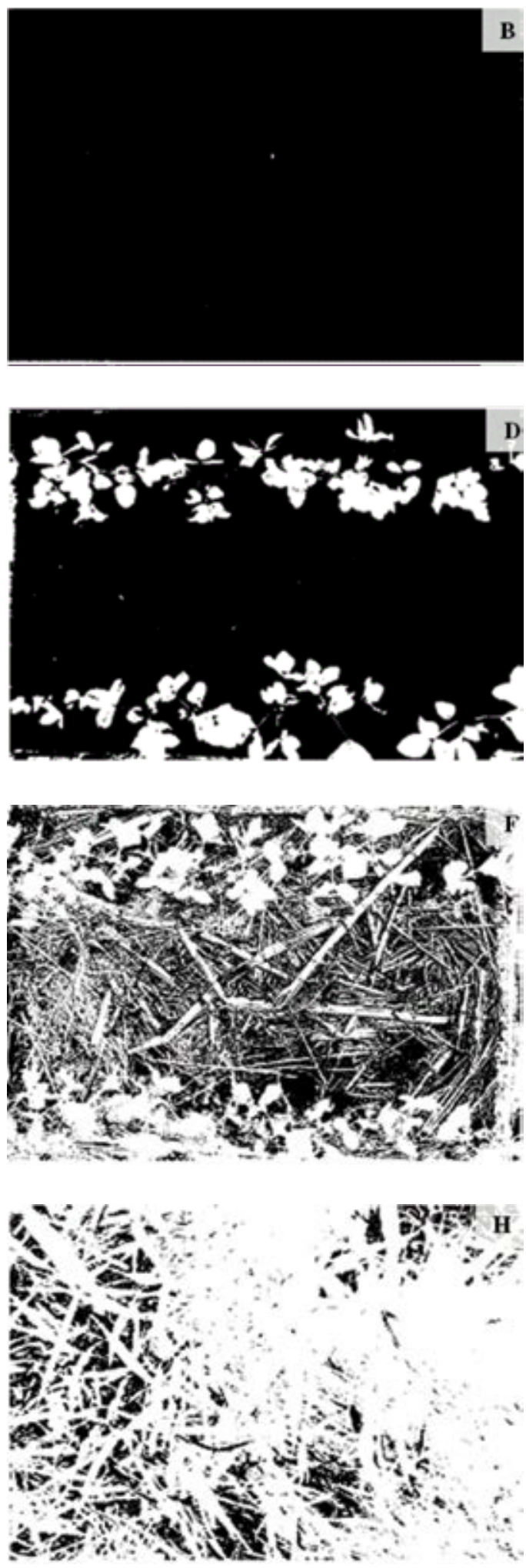

Figura 1. Imagens digitais $(A, C, E, G)$ e binárias $(B, D, F, H)$, respectivamente, para a estimativa do índice de cobertura vegetal, aos seguintes sistemas de cultivo: A, no solo exposto; $\mathrm{C}$, soja cultivada, com preparo convencional; E, soja em plantio direto; e G, pastagem estabelecida. As imagens foram tomadas 20 dias após a semeadura da cultura da soja. 
maior selamento da superfície. No entanto, as perdas de solo quantificadas no PA foram, em média, 2,55 vezes menores do que no sistema $\mathrm{PD}$, ao longo do período estudado, e aos 100 DAS se observaram perdas de solo no PD superiores a 8,9 vezes às perda no PA. Em cada época estudada, observaram-se diferenças (Scott Knott, $\mathrm{p}<5 \%$ ), apenas aos 40 e 80 DAS, entre o solo exposto e a soja sob preparo convencional de solo, o que mostra o efeito do preparo do solo e da cobertura vegetal no processo erosivo.

Quanto ao escoamento superficial, constatou-se diferença entre SE e PC apenas aos 80 DAS, com a diminuição do volume escoado em PC, ocasionado pela maior cobertura vegetal nesse sistema. Nos sistemas PD e PA, a diferença a partir dos 60 DAS é associada ao aumento do escoamento superficial em PA (Figura 3 B),
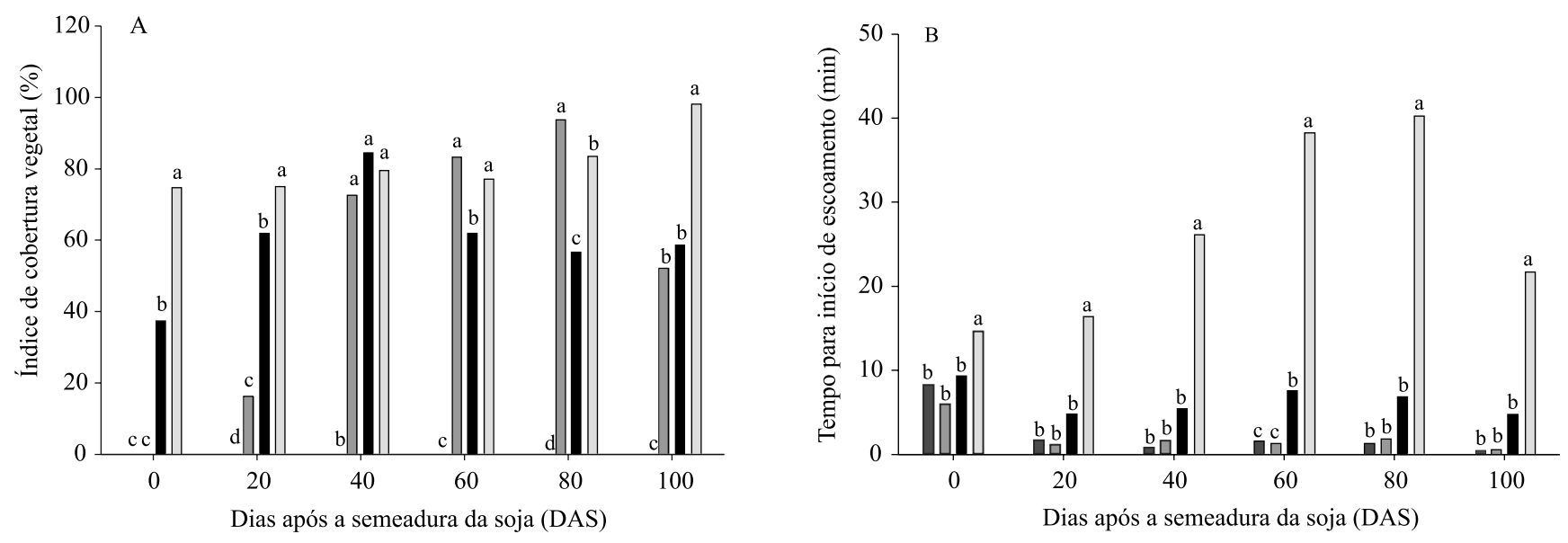

Figura 2. Índice de cobertura vegetal (ICV, A) e tempo para o início de escoamento em superfície (B), nos sistemas de cultivo em área de Argissolo Vermelho distrófico, por época de avaliação. SE, solo exposto; PC, soja cultivada, com preparo convencional do solo; PD, soja cultivada em plantio direto; e PA, pastagem estabelecida, sem pisoteio animal. Médias seguidas por letras iguais por época, não diferem pelo teste Scott-Knott, a 5\% de probabilidade.
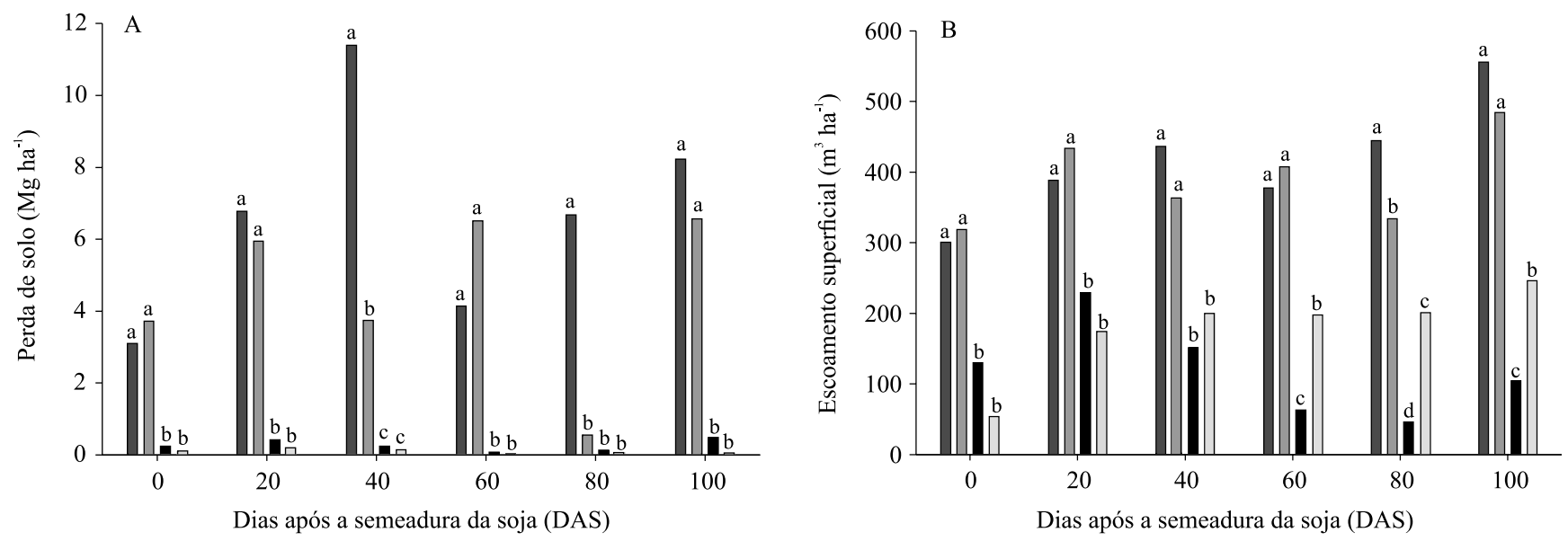

$\square \mathrm{SE} \square \mathrm{PC} \square \mathrm{PD} \square \mathrm{PA}$

Figura 3. Perda de solo (A) e escoamento superficial da água (B), por época de avaliação, nos sistemas de cultivo em Argissolo Vermelho distrófico. SE, solo exposto; PC, soja cultivada, com preparo convencional do solo; PD, soja cultivada em plantio direto; e PA, pastagem estabelecida, sem pisoteio animal. Médias seguidas por letras iguais por época, não diferem pelo teste Scott-Knott, a 5\% de probabilidade. 
resultante da quantidade de massa vegetal e arquitetura das gramíneas, que, por meio das folhas e colmos, podem ter favorecido o fluxo de água em direção à base das plantas. Em relação aos sistemas SE e PC, as perdas de água na pastagem são 125,5 e 96,6\% menores, respectivamente, aos 100 DAS. Nesta mesma avaliação, as perdas de solo nos tratamentos SE e PC, quando comparadas ao plantio direto, foram 431,2 e 361,2\% maiores, respectivamente. O aumento do escoamento superficial de água aos 100 DAS, em relação à etapa anterior, é justificado pelo menor índice de cobertura no tratamento $\mathrm{PC}$, em consequência da dessecação da soja, associada à umidade anterior aos testes. Em SE, condições da superfície, como menor rugosidade e selamento superficial, justificam as maiores perdas de água.

A combinação de época e sistema de cultivo interfere no processo erosivo (Scott Knott, $\mathrm{p}<0,05$ ), considerando-se o incremento da cobertura vegetal e o preparo do solo. Em SE e PC, a formação do selamento superficial e a diminuição da rugosidade do solo, com as subsequentes chuvas aplicadas, favoreceram $\mathrm{o}$ aumento das perdas de solo e água (Panachuki et al., 2011). Em PC, a partir dos 40 DAS, o aumento da cobertura vegetal da soja interferiu nas perdas de solo, reduzindo-as em comparação às primeiras etapas e, consequentemente, aos 80 DAS obteve-se menor perda de solo, quando a cobertura do solo foi máxima. Embora alguns estudos apontem que o escoamento superficial é muito influenciado por sistemas de preparo do solo, no presente trabalho constatou-se maior influência das práticas de manejo sobre as perdas de solo do que no escoamento de água em superfície. Engel et al. (2009) constataram que o escoamento superficial é resultante tanto das condições de superfície do solo (cobertura vegetal, cobertura do dossel ou rugosidade ao acaso) quanto das condições físicas do solo e do sistema de preparo que, em subsuperfície, provocam relativo grau de compactação, além de alterar a porosidade interna das camadas superficiais do solo. Entre os fatores que afetam o processo erosivo e o tempo inicial para escoamento em superfície destacaram-se, no presente estudo, as condições de preparo do solo, rugosidade da superfície do terreno, tipo e quantidade da cobertura vegetal sobre o solo, a alteração das propriedades físicas do solo e o teor de umidade do solo antecedente às chuvas.

\section{Conclusões}

1. A redução da erosão do solo sob cultivo de soja está associada à adoção de sistemas de cultivo que não revolvam o solo e à elevada cobertura vegetal.

2. Aredução do índice de cobertura vegetal, associada ao revolvimento do solo, acelera a desagregação de suas partículas pela ação de chuvas.

3 . As pastagens, quando adequadamente manejadas, proporcionam elevada cobertura ao solo, favorecem maior agregação de suas partículas e, consequentemente, reduzem a suscetibilidade do solo ao processo erosivo.

4. Em áreas sob preparo convencional e baixa cobertura vegetal da superfície do solo, a desagregação do solo e o processo erosivo ocorrem com a mesma intensidade que em áreas com solo exposto.

\section{Agradecimentos}

Ao Conselho Nacional de Desenvolvimento Científico e Tecnológico $(\mathrm{CNPq})$, pela concessão de bolsa; à Universidade Federal Rural do Rio de Janeiro (UFRRJ) e à Universidade Estadual de Mato Grosso do Sul (UEMS), pela disponibilização de estrutura.

\section{Referências}

ALVES SOBRINHO, T.; GÓMEZ-MACPHERSON, H.; GÓMEZ, J.A. A portable integrated rainfall and overland flow simulator. Soil Use and Management, v.24, p.163-170, 2008. DOI: 10.1111/j.1475-2743.2008.00150.x.

AMARAL, A.J. do; BERTOL, I.; COGO, N.P.; BARBOSA, F.T. Redução da erosão hídrica em três sistemas de manejo do solo em um Cambissolo Húmico da região do Planalto Sul-Catarinense. Revista Brasileira de Ciência do Solo, v.32, p.2145-2155, 2008. DOI: $10.1590 / \mathrm{S} 0100-06832008000500035$.

CENSO agropecuário 2006: resultados preliminares. Rio de Janeiro: Instituto Brasileiro de Geografia e Estatística, 2007. 142p.

CLAESSEN, M.E.C. (Org.). Manual de métodos de análise de solo. 2.ed. Rio de Janeiro: Embrapa-CNPS, 1997. 212p.

COGO, N.P.; MOLDENHAUER, W.C.; FOSTER, G.R. Soil loss reductions from conservation tillage practices. Soil Science Society of America Journal, v.48, p.368-373, 1984. DOI: 10.2136/ sssaj 1984.03615995004800020029x.

CORSINI, P.C.; FERRAUDO, A.S. Efeitos de sistemas de cultivo na densidade e macroporosidade do solo e no desenvolvimento radicular do milho em Latossolo Roxo. Pesquisa Agropecuária Brasileira, v.34, p.289-298, 1999. DOI: 10.1590/S0100204X1999000200017. 
CRUZ, E.S.; CARVALHO, D.F.; VARELLA, C.A.A.; SILVA, L.D.B.; SOUZA, W.J.; PINTO, F.A.C. Comparação de classificadores de imagens digitais na determinação da cobertura do solo. Engenharia Agrícola, v.28, p.237-244, 2008. DOI: 10.1590/S0100-69162008000200004.

DALLA ROSA, J.; COOPER, M.; DARBOUX, F.; MEDEIROS, J.C. Soil roughness evolution in different tillage systems under simulated rainfall using a semivariogram-based index. Soil and Tillage Research, v.124, p.226-232, 2012. DOI: 10.1016/j. still.2012.06.001.

ENGEL, F.L.; BERTOL, I.; RITTER, S.R.; PAZ GONZÁLEZ, A.; PAZ-FERREIRO, J.; VIDAL VÁZQUEZ, E. Soil erosion under simulated rainfall in relation to phenological stages of soybeans and tillage methods in Lages, SC, Brazil. Soil and Tillage Research, v.103, p.216-221, 2009. DOI: 10.1016/j.still.2008.05.017.

HUANG, J.; WU, P.; ZHAO, X. Effects of rainfall intensity, underlying surface and slope gradient on soil infiltration under simulated rainfall experiments. Catena, v.104, p.93-102, 2013. DOI: $10.1016 /$ j.catena.2012.10.013.

IBGE. INSTITUTO BRASILEIRO DE GEOGRAFIA E ESTATÍSTICA. Levantamento sistemático da produção agrícola. 2015. Disponível em: <http://www.ibge.gov.br/home/ estatistica/indicadores/agropecuaria/lspa/default.shtm $>$. Acesso em: 14 dez. 2015.

LAPOLA, D.M.; MARTINELLI, L.A.; PERES, C.A.; OMETTO, J.P.H.B.; FERREIRA, M.E.; NOBRE, C.A.; AGUIAR, A.P.D.; BUSTAMANTE, M.M.C.; CARDOSO, M.F.; COSTA, M.H.; JOLY, C.A.; LEITE, C.C.; MOUTINHO, P.; SAMPAIO, G.; STRASSBURG, B.B.N.; VIEIRA, I.C. Pervasive transition of the Brazilian land-use system. Nature Climate Change, v.4, p.27-35, 2013. DOI: $10.1038 /$ nclimate2056.

NACINOVIC, M.G.G.; MAHLER, C.F.; AVELAR, A. de S. Soil erosion as a function of different agricultural land use in Rio de Janeiro. Soil and Tillage Research, v.144, p.164-173, 2014. DOI: 10.1016/j.still.2014.07.002.

PANACHUKI, E.; BERTOL, I.; ALVES SOBRINHO, T.; OLIVEIRA, P.T.S. de; RODRIGUES, D.B.B. Perdas de solo e de água e infiltração de água em Latossolo Vermelho sob sistemas de manejo. Revista Brasileira de Ciência do Solo, v.35, p.17771785, 2011. DOI: 10.1590/S0100-06832011000500032.

PANACHUKI, E.; BERTOL, I.; ALVES SOBRINHO, T.; VITORINO, A.C.T.; SOUZA, C.M.A. de; URCHEI, M.A. Rugosidade da superfície do solo sob diferentes sistemas de manejo e influenciada por chuva artificial. Revista Brasileira de Ciência do Solo, v.34, p.443-451, 2010. DOI: 10.1590/S010006832010000200018.

PANAGOS, P.; BORRELLI, P.; MEUSBURGER, K.; ALEWELL, C.; LUGATO, E.; MONTANARELLA, L. Estimating the soil erosion cover-management factor at the European scale. Land Use Policy, v.48, p.38-50, 2015. DOI: 10.1016/j. landusepol.2015.05.021.

PRADO, E.A.F. do; VITORINO, A.C.T.; OLIVEIRA, W.H. de; ESPINDOLA, D.L.P.; ARANTES, H.P. Índice de dispersão de agregados de um Latossolo Vermelho distroférrico cultivado com cana sob aplicação de vinhaça. Semina: Ciências Agrárias, v.35, p.2347-2356, 2014. DOI: 10.5433/1679-0359.2014v35n4Suplp2347.

REINERT, D.J.; ALBUQUERQUE, J.A.; REICHERT, J.M.; AITA, C.; ANDRADA, M.M.C. Limites críticos de densidade do solo para o crescimento de raízes de plantas de cobertura em Argissolo Vermelho. Revista Brasileira de Ciência do Solo, v.32, p.1805-1816, 2008. DOI: 10.1590/S010006832008000500002 .

RIES, J.B.; SEEGER, M.; ISERLOH, T.; WISTORF, S.; FISTER, W. Calibration of simulated rainfall characteristics for the study of soil erosion of agricultural land. Soil and Tillage Research, v.106, p.109-116, 2009. DOI: 10.1016/j.still.2009.07.005.

SANTOS, M.A. do N. dos; PANACHUKI, E.; ALVES SOBRINHO, T.; OLIVEIRA, P.T.S. de; RODRIGUES, D.B.B. Water infiltration in an Ultisol after cultivation of common bean. Revista Brasileira de Ciência do Solo, v.38, p.1143-1152, 2014. DOI: 10.1590/S0100-06832014000500026.

SCHIAVO, J.A.; PEREIRA, M.G.; MIRANDA, L.P.M. de; DIAS NETO, A.H.; FONTANA, A. Caracterização e classificação de solos desenvolvidos de arenitos da formação Aquidauana-MS. Revista Brasileira de Ciência do Solo, v.4, p.881-889, 2010. DOI: 10.1590/S0100-06832010000300029.

SERAFIM, M.E.; VITORINO, A.C.T.; PEIXOTO, P.P.P.; SOUZA, C.M.A.; CARVALHO, D.F. de. Intervalo hídrico ótimo em um Latossolo Vermelho distroférrico sob diferentes sistemas de produção. Engenharia Agrícola, v.28, p.654-665, 2008. DOI: 10.1590/S0100-69162008000400005.

SOUZA, Z.M. de; PRADO, R. de M.; PAIXÃO, A.C.S.; CESARIN, L.G. Sistemas de colheita e manejo da palhada de cana-de-açúcar. Pesquisa Agropecuária Brasileira, v.40, p.271-278, 2005. DOI: 10.1590/S0100-204X2005000300011.

WANG, X.; ZHAO, X.; ZHANG, Z.; YI, L.; ZUO, L.; WEN, Q.; LIU, F.; XU, J.; HU, S.; LIU, B. Assessment of soil erosion change and its relationships with land use/cover change in China from the end of the 1980s to 2010. Catena, v.137, p.256-268, 2016. DOI: 10.1016/j.catena.2015.10.004.

ZIMMER, A.H.; MACEDO, M.C.M.; KICHEL, A.N.; ALMEIDA, R.G. de. Degradação, recuperação e renovação de pastagens. Embrapa Gado de Corte, 2012. 42p. (Embrapa Gado de Corte. Documentos, 189).

Recebido em 29 de agosto de 2015 e aprovado em 14 de dezembro de 2015 